\title{
Competencia clínica de médicos guatemaltecos y mexicanos para el manejo de la disfunción familiar
}

\author{
Carlos Enrique Cabrera-Pivaral',2, María de Jesús Orozco-Valerio², Alfredo Celis-de la Rosa²,
} María de los Ángeles Covarrubias-Bermúdez y Marco Antonio Zavala-González ${ }^{3}$

${ }^{1}$ Unidad de Investigación, Instituto Guatemalteco de Seguridad Social, Ciudad de Guatemala, Guatemala; ${ }^{2}$ División de Disciplinas para el Desarrollo, Promoción y Preservación de la Salud; ${ }^{3}$ Doctorado en Ciencias de la Salud Pública, Departamento de Salud Pública. Centro Universitario de Ciencias de la Salud, Universidad de Guadalajara, Guadalajara, Jal., México

\begin{abstract}
Resumen
Objetivo: Evaluar el nivel de competencia clínica de médicos mexicanos y guatemaltecos para manejar la disfunción familiar. Método: Diseño transversal comparativo en cuatro unidades de atención primara de la ciudad de Guadalajara, México, y cuatro de Ciudad de Guatemala, Guatemala, según un muestreo propositivo; participaron 117 y 100 médicos, respectivamente. Competencia clínica evaluada mediante instrumento validado integrado por 187 reactivos. Se realizó análisis estadístico descriptivo e inferencial no paramétrico. Resultados: El porcentaje de médicos mexicanos con competencia clínica alta fue del $13.7 \%$, media $53 \%$, baja $24.8 \%$ y definida por el azar $8.5 \%$. Para los médicos guatemaltecos, el $14 \%$ fue alto, el $63 \%$ medio y el $23 \%$ definido por el azar. No hubo diferencias estadísticamente significativas entre unidades médicas por país, pero ni entre las medianas de los mexicanos $(0.55)$ y de los guatemaltecos $(0.55)(p=0.02)$. Conclusión: La competencia clínica alta entre los médicos mexicanos aumentó es igual que en los guatemaltecos.
\end{abstract}

PALABRAS CLAVE: Salud de la familia. Medicina familiar y comunitaria. Médicos familiares. Competencia clínica.

\begin{abstract}
Objective: To evaluate the clinical competence of Mexican and Guatemalan physicians to management the family dysfunction. Methods: Cross comparative study in four care units first in Guadalajara, Mexico, and four in Guatemala, Guatemala, based on a purposeful sampling, involving 117 and 100 physicians, respectively. Clinical competence evaluated by validated instrument integrated for 187 items. Non-parametric descriptive and inferential statistical analysis was performed. Results: The percentage of Mexican physicians with high clinical competence was $13.7 \%$, medium $53 \%$, low $24.8 \%$ and defined by random $8.5 \%$. For the Guatemalan physicians' $14 \%$ was high, average $63 \%$, and $23 \%$ defined by random. There were no statistically significant differences between healthcare country units, but between the medium of Mexicans (0.55) and Guatemalans (0.55) $(p=0.02)$. Conclusion: The proportion of the high clinical competency of Mexican physicians' was as Guatemalans.
\end{abstract}

KEY WORDS: Family health. Family practice. Physicians'family. Clinical competence.

\section{Correspondencia:}

María de los Ángeles Covarrubias-Bermúdez

Sierra Mojada, 950, Puerta 1, Edificio N, Planta Alta

Col. Lomas de Independencia

Fecha de recepción: 13-07-2016

C.P. 44240 , Guadalajara, Jal., México

Fecha de aceptación: 18-08-2016

E-mail: angelescovarrubias@hotmail.com

DOI:10.24875/GMM.17002659

Gac Med Mex. 2017;153:683-687

Contents available at PubMed www.gacetamedicademexico.com 


\section{Introducción}

La familia es considerada un indicador de salud ${ }^{1} y$ un punto central para la atención integral de la salud en los individuos ${ }^{2}$. Específicamente, manejar la disfunción familiar es una estrategia para abordar problemas de salud ${ }^{3}$, debido a que esta es un factor de riesgo para la obesidad en niños ${ }^{4}$ y adultos mexicanos $^{5}$, se relaciona con un mal estado nutricio de colombianos geriátricos ${ }^{6}$ y la inaccesibilidad de los niños a los alimentos ${ }^{7}$, se asocia a ideación e intento suicida en adolescentes colombianos y chilenos ${ }^{8,9}$, y es uno de los factores de riesgo más importantes del suicidio en la población de América Latina y el Caribe $^{10,11}$.

En este sentido, la competencia clínica del médico para prevenir, diagnosticar y tratar la disfuncionalidad familiar tiene suma importancia y por ende resulta necesario evaluarla ${ }^{3}$. Algunos autores han mostrado que dicha competencia suele ser baja en el personal médico; por ejemplo, en Cuba, se evaluó la competencia de 25 residentes de medicina para la atención a la familia y solo el $11,8 \%$ aprobó más del $70 \%$ de la evaluación ${ }^{12}$. En México, se mostró que la competencia de estudiantes de medicina de distinto grado fue muy baja $\mathrm{a}^{13}$, y otro estudio que evaluó la competencia de 200 médicos mostró que solo el $3 \%$ obtuvo un puntaje alto para manejar la disfunción familiar ${ }^{14}$.

Los estudios anteriores muestran un conocimiento significativo, empero es importante realizar estudios de seguimiento debido a que la competencia clínica del médico es un indicador indirecto de la calidad de la atención que proporciona al paciente ${ }^{15}$. También es importante comparar dicha competencia en contextos distintos para identificar elementos comunes. Debido a que Guatemala y México tienen un sistema de salud similar ${ }^{16,17}$ y existe cooperación por parte de colegas en ambos países, el objeto de este estudio es evaluar la competencia clínica de médicos mexicanos y guatemaltecos para manejar la disfunción familiar.

\section{Método}

Se llevó a cabo un estudio transversal comparativo (Fig. 1) en cuatro clínicas de la Ciudad de Guadalajara, Jalisco, México, y otras cuatro de la Ciudad de Guatemala, Guatemala, durante el año 2015. Para la elección de la muestra se hizo un muestreo por conglomerados de cuatro unidades clínicas de primer nivel de atención en cada una de las ciudades, en las cuales se localizaron 250 médicos guatemaltecos y 450 mexicanos. Mediante un muestreo propositivo se obtuvo una muestra de 117 médicos en Guadalajara y 100 en Guatemala.

Se incluyeron todos los médicos que aceptaron participar en el estudio. Se descartaron aquellos que laboraban en el área de urgencias o en turno nocturno. $Y$ se eliminaron los cuestionarios que no fueron respondidos completamente.

La variable de estudio fue la competencia clínica de los participantes para manejar la disfunción familiar, considerando los siguientes indicadores: a) identificación de factores de riesgo, b) integración diagnóstica, y c) identificación de propuestas y orientación.

Para evaluar la competencia clínica se utilizó un instrumento previamente validado ${ }^{14}$, constituido por 187 reactivos basados en casos clínicos problematizados. Las opciones de respuesta fueron «verdadero», «falso» y «no sé», cuyos valores fueron de 1 para respuesta correcta, -1 para incorrecta y 0 para «no sé». El valor teórico máximo fue de 187 puntos entre todos los indicadores considerados. Adicionalmente se incluyeron variables académicas y sociodemográficas para caracterizar al grupo de estudio.

Basándose en lo referido por Viniegra, et al..$^{15}$ se consideraron los siguientes grados para medir la competencia clínica: a) definidos por el azar $<60$ puntos, b) nivel bajo $60-99$, c) nivel medio 100-149, y d) nivel alto $>140$, con una fiabilidad del $90 \%$ de acuerdo con la prueba de Kuder-Richardson.

La evaluación del instrumento fue realizada por una persona externa al estudio. Mediante estadística descriptiva se obtuvieron las frecuencias y porcentajes en relación a las características de los participantes y su nivel de competencia. Además, se obtuvo la diferencia de la competencia entre grupos del mismo país comparando las medianas del nivel de competencia clínica utilizando la prueba de Kruskal-Wallis, y finalmente se utilizó la $U$ de Mann-Whitney para comparar la mediana global de la competencia clínica de los médicos según elpaís de origen. El nivel de confianza para las estadísticas fue del95\% ( $p \leq 0.05)$, utilizando Epi Info ${ }^{\odot}$ versión 7.1.5.2 para Windows ${ }^{\odot}$.

Como parte de las consideraciones éticas, se tomó en cuenta lo establecido en el Reglamento de la Ley de Salud en Materia de Investigación para la Salud ${ }^{18}$ y el Código de Salud de la República de Guatemala ${ }^{19}$, según los cuales este estudio es considerado sin riesgo, ya que no se intervino a la población y además se consiguió el consentimiento verbal de los participantes y se les aseguró el anonimato. 


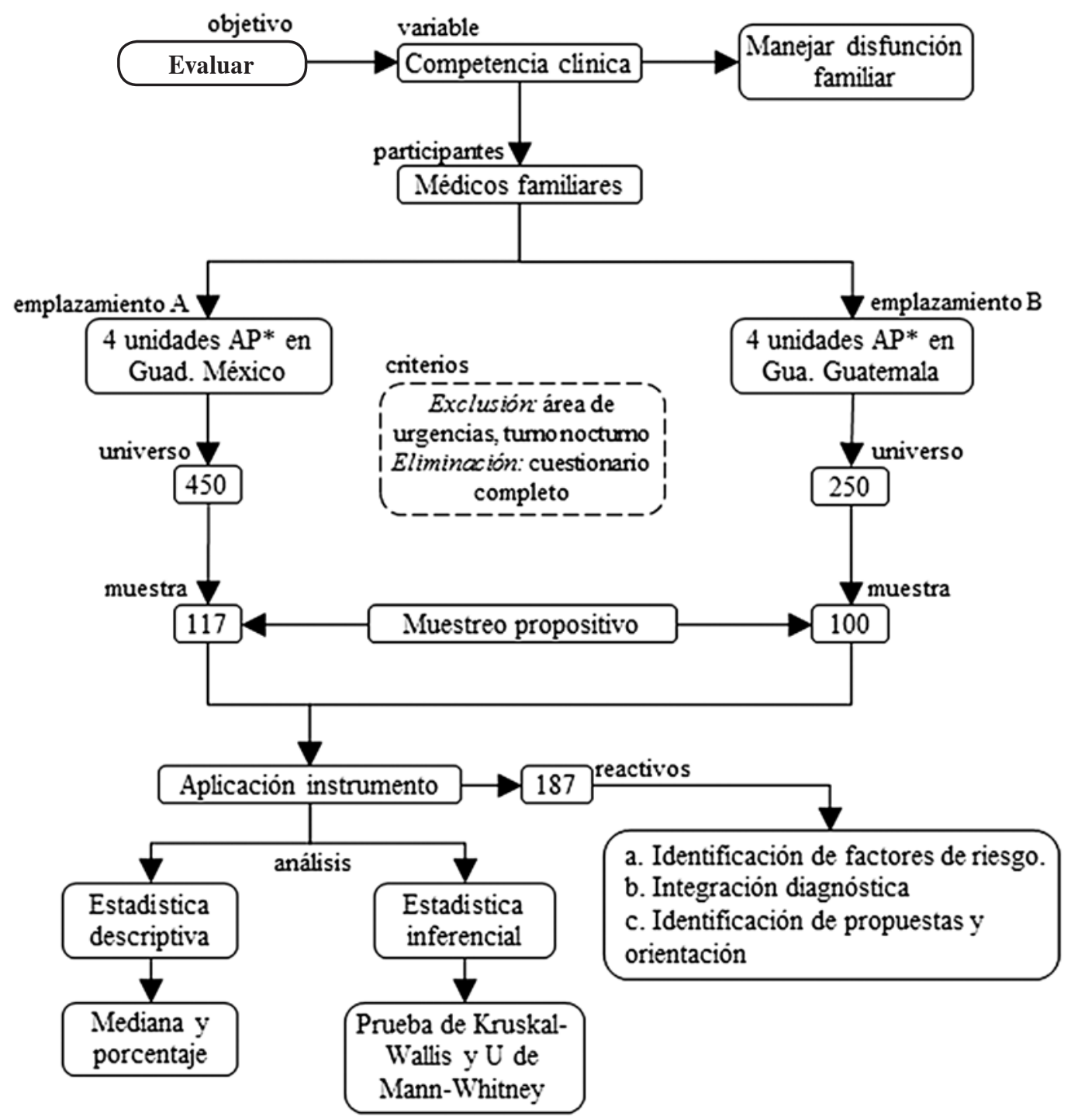

Figura 1. Proceso metodológico del estudio (fuente:elaboración propia).

\section{Resultados}

Las características de los grupos estudiados se muestran en la tabla 1.

La evaluación de la competencia clínica para tratar la disfunción familiar mostró que el $13.7 \%$ de los mexicanos y el $14 \%$ de los guatemaltecos obtuvieron una puntuación alta; en el mismo orden, el 53 y el $63 \%$ un nivel medio, el 24.8 y el $23 \%$ un nivel bajo, y el $8.5 \%$ un nivel definido por el azar solo para los mexicanos (Tabla 2).

Al comparar las unidades médicas de Guadalajara no se encontraron diferencias estadísticamente significativas en relación con su nivel de competencia clínica, al igual que en las unidades de Guatemala (Tabla 3).
La comparación entre las medianas globales de cada muestra reveló una diferencia estadísticamente significativa (Tabla 3). La mediana del grupo de Guadalajara fue mayor que la de Guatemala ( $p>0.05)$.

\section{Discusión}

Este estudio representa una medición actual de la competencia clínica de médicos mexicanos para manejar la disfunción familiar. En el caso de Guatemala, aporta nuevo conocimiento debido a que no se había explorado en este país.

Los resultados, aunque similares, no son comparables con los de otros estudios ${ }^{12,13}$ existentes porque los indicadores, los métodos y los participantes considerados por los autores son distintos. Sin embargo, son 
Tabla 1. Características demográficas, académicas y laborales de los médicos

\begin{tabular}{|c|c|c|c|c|}
\hline \multirow[t]{2}{*}{ Variables } & \multicolumn{2}{|c|}{$\begin{array}{c}\text { Guadalajara } \\
\qquad(N=117)\end{array}$} & \multicolumn{2}{|c|}{$\begin{array}{l}\text { Guatemala } \\
(N=100)\end{array}$} \\
\hline & $f$ & $\%$ & $f$ & $\%$ \\
\hline $\begin{array}{l}\text { Sexo } \\
\text { Mujer } \\
\text { Hombre }\end{array}$ & $\begin{array}{l}47 \\
70\end{array}$ & $\begin{array}{l}40.2 \\
59.8\end{array}$ & $\begin{array}{l}52 \\
48\end{array}$ & $\begin{array}{l}52.0 \\
48.0\end{array}$ \\
\hline $\begin{array}{l}\text { Contratación } \\
\text { De base } \\
\text { Eventual }\end{array}$ & $\begin{array}{l}98 \\
19\end{array}$ & $\begin{array}{l}83.8 \\
16.2\end{array}$ & $\begin{array}{l}56 \\
44\end{array}$ & $\begin{array}{l}56.0 \\
44.0\end{array}$ \\
\hline $\begin{array}{l}\text { Antigüedad laboral (años) } \\
\quad<10 \\
10-19 \\
>20\end{array}$ & $\begin{array}{l}19 \\
56 \\
42\end{array}$ & $\begin{array}{l}16.2 \\
47.9 \\
35.9\end{array}$ & $\begin{array}{l}42 \\
24 \\
34\end{array}$ & $\begin{array}{l}42.0 \\
24.0 \\
34.0\end{array}$ \\
\hline $\begin{array}{l}\text { Experiencia (años) } \\
\quad<10 \\
10-19 \\
>20\end{array}$ & $\begin{array}{l}33 \\
42 \\
42\end{array}$ & $\begin{array}{l}28.2 \\
35.9 \\
35.9\end{array}$ & $\begin{array}{l}56 \\
32 \\
12\end{array}$ & $\begin{array}{l}56.0 \\
32.0 \\
12.0\end{array}$ \\
\hline $\begin{array}{l}\text { Turno } \\
\text { Matutino } \\
\text { Vespertino }\end{array}$ & $\begin{array}{l}61 \\
56\end{array}$ & $\begin{array}{l}52.1 \\
47.9\end{array}$ & $\begin{array}{l}68 \\
32\end{array}$ & $\begin{array}{l}68.0 \\
32.0\end{array}$ \\
\hline
\end{tabular}

Tabla 2. Nivel de competencia clínica de los médicos por estratos

\begin{tabular}{lcccccc}
\hline \multirow{2}{*}{$\begin{array}{l}\text { Nivel de } \\
\text { competencia }\end{array}$} & $\begin{array}{l}\text { Intervalo de } \\
\text { puntuación }\end{array}$ & \multicolumn{2}{c}{ Guadalajara } & & \multicolumn{2}{c}{ Guatemala } \\
\cline { 3 - 4 } \cline { 6 - 7 } & & $\boldsymbol{f}$ & $\%$ & & $\boldsymbol{f}$ & $\%$ \\
\hline $\begin{array}{l}\text { Definido por el } \\
\text { azar }\end{array}$ & $<$ & 10 & 8.5 & & 0 & 0.0 \\
Bajo & $9-16$ & 29 & 24.8 & & 23 & 23.0 \\
Medio & $17-24$ & 62 & 53.0 & & 63 & 63.0 \\
Alto & 25 & 16 & 13.7 & & 14 & 14.0 \\
Total & & 117 & 100.0 & & 100 & 100.0 \\
\hline
\end{tabular}

Fuente:instrumento aplicado a médicos familiares de atención primaria de Guadalajara y Guatemala. equiparables a los presentados por Cabrera-Pivaral, et al. ${ }^{14}$, quienes en su estudio muestran que el nivel de competencia clínica alta de los médicos mexicanos aumentó un $10.3 \%$ en los últimos 9 años. Es posible que este resultado se deba a la implementación del Programa Sectorial de Salud ${ }^{20}$, que enfatiza la necesidad de atender la salud en consonancia con el género, la cultura y los estilos de vida, donde el trabajo con la familia es básico.

Por otro lado, aunque los mexicanos mostraron una ventaja significativa sobre los guatemaltecos respecto a la competencia clínica alta, en ambos contextos el nivel de competencia resultó insuficiente, debido a que el 86.3 y el $86 \%$, respectivamente, obtuvieron puntajes que los posicionaron en los niveles medio, bajo y definido por el azar. Este resultado puede deberse a la carencia de programas educativos orientados a la atención primaria y el trabajo interdisciplinario, así como al número reducido de universidades que acrediten la calidad de sus servicios ${ }^{21}$.

Para el caso específico de Guatemala, reportes nacionales muestran que el sistema de salud se mantiene dentro de un modelo de atención biomédico, dado que trabajadores sociales y psicólogos actúan solo de manera complementaria, circunstancia que dificulta al médico atender a los pacientes desde el punto de vista de la familia ${ }^{22}$. De la misma manera, aunque la atención primaria es prioritaria, las acciones de salud son mayoritariamente para el diagnóstico, el tratamiento y la rehabilitación ${ }^{23}$, por lo cual la atención a la disfuncionalidad familiar muchas veces es desplazada.

Es necesario realizar nuevos estudios que incluyan variables organizativas y del ambiente laboral para explicar las diferencias entre el nivel de competencia de los grupos considerados en este estudio.

Tabla 3. Nivel de competencia clínica de los médicos por unidad médica y ciudad

\begin{tabular}{|c|c|c|c|c|c|c|c|}
\hline \multirow[t]{2}{*}{ Unidad médica } & \multicolumn{3}{|c|}{ Guadalajara } & \multicolumn{3}{|c|}{ Guatemala } & \multirow[t]{2}{*}{$\mathbf{p}^{\dagger}$} \\
\hline & $\mathrm{N}$ & Mediana & Intervalo & $\mathbf{n}$ & Mediana & Intervalo & \\
\hline$A$ & 30 & 19 & $6-24$ & 21 & 15 & $4-19$ & 0.02 \\
\hline B & 27 & 15 & $12-26$ & 24 & 12 & $8-21$ & 0.01 \\
\hline C & 29 & 18 & $8-25$ & 26 & 14 & $6-19$ & 0.04 \\
\hline $\mathrm{D}$ & 31 & 17 & $12-27$ & 29 & 12 & $9-21$ & 0.01 \\
\hline Total & 117 & 15 & $6-27$ & 100 & 12 & $4-21$ & 0.02 \\
\hline$p^{*}$ & 0.55 & & & 0.55 & & & - \\
\hline
\end{tabular}


En cuanto a la representatividad de la muestra, se considera adecuada puesto quese evaluó la competencia clínica de un número de participantes que sobrepasó lo mínimo requerido para un estudio de tipo descriptivo de variables cualitativas en universos finitos, $\mathrm{n}=\left(\mathrm{NZ} \mathrm{Z}^{\wedge} 2 \mathrm{pq}\right) /\left(\mathrm{d}^{\wedge} 2(\mathrm{~N}-1)+\mathrm{Z}^{\wedge} 2 \mathrm{pq}\right)$, considerando, por ejemplo, en el universo del contexto mexicano de $N=450$, una $p=0.88(q=0.12)$, esto con base en una prevalencia de la competencia clínica de nivel medio a definido por el azar del $88 \%$ en los médicos mexicanos ${ }^{14}$.

Sin embargo, como limitación del estudio se considera que la composición de la muestra mediante muestreo propositivo pudo generar un sesgo de selección, debido a que la motivación de los médicos para participar pudo ser la autopercepción de un nivel de dominio alto del tema, en cuyo caso los resultados estarían sobreestimados.

No obstante, la heterogeneidad de los resultados pudo mostrar que resulta necesario evaluar la competencia clínica de los médicos para manejar la disfunción familiar, ya que es un indicador de problemas en la práctica de los médicos, mismos que obstaculizan la atención a la salud en concordancia con lo planteado por los gobiernos de México y Guatemala, los cuales se proponen establecer estrategias de acción basadas en las necesidades de la familia y la comunidad ${ }^{20,24}$.

\section{Bibliografía}

1. Organización Mundial de la Salud. Índices estadísticos de la salud de la familia. Washington, D.C.: Organización Mundial de la Salud; 1976 (Consultado el 1 de junio de 2016.)Disponible en: http://apps.who.int/iris/ bitstream/10665/40938/1/WHO_TRS_587_spa.pdf.

2. Herrera Santí, PM. La familia funcional y disfuncional, un indicador de salud. Rev Cubana Med Gen Intergr. 1997;13(6):591-9. Disponible en: http://scielo. sld.cu/scielo.php?script=sci_arttext\&pid=S0864-21251997000600013\&lng=es.

3. Garza-Elizondo T. Dimensiones de la funcionalidad familiar a evaluar en la entrevista familiar. En: Trabajo con familias: abordaje médico e integral. 3. ${ }^{a}$ ed. México: Garza \& Gutiérrez; 2015. 97-104.

4. González-Rico JL, Vásquez-Garibay EM, Cabrera-Pivaral CE, et al. La disfunción familiar como factor de riesgo para obesidad en escolares mexicanos. Rev Med Inst Mex Seguro Soc. 2012;50:127-34.
5. Jaramillo-Sánchez R. Disminución de peso y funcionalidad familiar en sujetos con obesidad y sobrepeso. Rev Med Inst Mex Seguro Soc. 2012;50:487-92.

6. Carrazco-Peña KB, Tene CE, Elizalde AM. Disfunción familiar y desnutrición en el anciano. Rev Med Inst Mex Seguro Soc. 2015;53:14-9.

7. Taborda-Restrepo PA, Pérez-Cano ME. Funcionalidad familiar, seguridad alimentaria y estado nutricional del programa departamental de complementación alimentaria de Antioquia. CES Medicina. 2011;25:6-19.

8. Von-Dessauer B, Ortiz P, Hinostroza T, et al. Intento de suicidio vía ingesta de fármacos en niños. RevChilPediatr.2011;82:42-8.

9. Álvarez-Latorre JM, Cañón-Castrillón JJ, Bernier-Ocampo LH, et al. Factor de riesgo suicida y factores asociados en adolescentes de una institución educativa de Palestina-Caldas. Arch Med. 2012;13:127-41.

10. Cañón-Buitrago SC. Factores de riesgo asociados a conductas suicidas en niños y adolescentes. Arch Med. 2011;11:62-7.

11. Teti G, Rebok F, Rojas SM, et al. Systematic review of risk factors for suicide and suicide attempt among psychiatric patients in Latin America and Caribbean. Rev Panam Salud Públ. 2014;36:124-33.

12. Rocha-Vázquez M, San Juan-Bosh MA. Evaluación de la competencia profesional de residentes de Medicina General Integral para la atención a la familia. Educ Med. 2010;13:41-6.

13. Dorado-Gaspar MP, Loría-Castellanos J, Guerrero-Martínez MB. Aptitud clínica hacia el estudio integral de la familia en residentes de medicina familiar. Arch Med Fam. 2006;3:169-74.

14. Cabrera-Pivaral CE, Rodríguez-Pérez I, González-Pérez G, et al. Aptitud clínica de los médicos familiares en la identificación de la disfunción familiar, en unidades de medicina familiar de Guadalajara, México. Salud Mental. 2006;29:40-6.

15. Viniegra $L$, Jiménez JL, Pérez Padilla JR. El desafío de la evaluación de la competencia clínica. Rev Invest Clin. 1991;43:87-95.

16. Gómez O, Sesma S, Becerril V, et al. Sistema de salud de México. Salud Publica Mex.2011;53:220-32.

17. Becerril-Montekio V, López-Dávila L. Sistema de salud de Guatemala. Salud Publica Mex.2011;53(Supl2):197-208.

18. Reglamento de la Ley general de salud en materia de investigación para la salud. México D.F.: Diario Oficial de la Federación de los Estados Unidos Mexicanos; 1986.

19. Código de salud. Decreto número 90-97. Ciudad de Guatemala: Diario Oficial de la Federación de la República de Guatemala; 1997.

20. Gobierno de la República. Programa Sectorial de Salud 2013-2018. México, D.F.: Diario Oficial de la Federación; 2013.

21. Organización Panamericana de la Salud. La renovación de la atención primaria de salud en las Américas. La formación en medicina orientada hacia la atención primaria de salud. Washington D.C.: Organización Panamericana de la Salud; 2008. (Consultado el 1 de junio de 2016.) Disponible en: http://www.nodo50.org/fadsp/archivos/APS-Formacion_ Medicina_Orientada_APS\%5B1\%5D.pdf.

22. Avila C, Bright R, Gutiérrez JC, et al.Análisis del Sistema de Salud 2015. Guatemala: Proyecto Health Finance and Governance. Abt Associates; 2015. (Consultado el 1 de junio de 2016.)Disponible en: https://www. usaid.gov/sites/default/files/documents/1862/Guatemala-Analisis-del-Sector-Publico-Salud-Esp-INFORME-COMPLETO-FINAL-Abr2016.pdf.

23. Estrada-Galindo G. El sistema de salud en Guatemala.Guatemala: Naciones Unidas; 2008. (Consultado el 1 de junio de 2016.)Disponible en: https://idl-bnc-idrc.dspacedirect.org/bitstream/handle/10625/36342/127653. pdf?sequence=1\&isAllowed=y.

24. Ministerio de Salud Pública y Asistencia Social. Diagnóstico Nacional de Salud. Guatemala: Ministerio de Salud Pública y Asistencia Social; 2012. (Consultado el 1 de junio de 2016.)Disponible en: http://www.mspas.gob. gt/images/files/cuentasnacionales/publicaciones/11MSPAS(2012)Salud-marzo.pdf. 